liability for autism [2]. Another population-based Swedish study based only on twins estimated a heritability of $80 \%$ [3].

\title{
References.
}

1. Sandin S, et al. JAMA. 2014 May 7;311(17):1770-7.

2. Schendel D, et al. JAMA. 2014 May 7;311(17):1738-9.

3. Lichtenstein P, et al. Am J Psychiatry. 2010 Nov;167(11):1357-63.

\section{PARENTAL OBESITY AND AUTISM SPECTRUM DISORDER}

Investigators from Oslo, Norway and other centers studied the associations among maternal prepregnancy BMI, paternal BMI, and the risk of autism spectrum disorder (ASD) in children. A study sample of 92909 children aged 4 to 13.1 (mean 7.4) years was derived from a population-based, prospective Norwegian Mother and Child Cohort Study. Among 419 children diagnosed with ASD at end of follow-up (2012), 162 were diagnosed with autistic disorder, 103 with Asperger disorder, and 154 with PDD. Maternal obesity (BMI >30) was only weakly associated with ASD risk, whereas paternal obesity was associated with an increased risk of autistic disorder (AD) and Asperger disorder. The risk of $\mathrm{AD}$ was $0.27 \%$ in children of obese fathers and $0.14 \%$ in children of fathers with normal weight $(\mathrm{BMI}<25)$. The risk of Asperger disorder was $0.38 \%$ in children (aged $>7$ years) of obese fathers and $0.18 \%$ in children of normal-weight fathers. The adjusted OR for AD was 1.73 and for Asperger disorder, 2.01. Parental obesity was not associated with PDD. (Suren P, Gunnes N, Roth C, et al. Parental obesity and risk of autism spectrum disorder. Pediatrics 2014 May 1;133(5):e1128-e1138).

COMMENTARY. Adverse effects of obesity in relation to childhood ASD concern both child and father. In a sample of 376 Oregon children with ASD, $18.1 \%$ of children met criteria for overweight and $17.0 \%$ met criteria for obesity [1].

Gluten-free casein-free ketogenic diet for autism and seizures. Pediatric neurologists at the Massachusetts General Hospital, Boston, MA, report a girl with autism and pubertal onset of seizures refractory to AEDs who benefited from treatment with a gluten-free casein-free ketogenic diet, with medium-chain triglycerides. Secondary benefits of the MCT diet included resolution of morbid obesity and improved cognition and behavior. The Childhood Autism Rating Scale score decreased from 49 (severe) to 17 (nonautistic); the child was essentially seizure free after 14 months on the diet; and a lengthy $3 \mathrm{~Hz}$ spike-wave EEG pattern improved, showing only occasional short discharges without clinical accompaniments [2]. Despite the limitations of a single case, this report seemed worthy of comment.

\section{References.}

1. Zuckerman KE, et al. J Autism Dev Disord. 2014 Feb 2. [Epub ahead of print]

2. Herbert MR, Buckley JA. J Child Neurol. 2013 Aug;28(8):975-82. 